\title{
Persönliches Wort
}

Ulrich Seibert kenne ich schon sehr lange und war schon früh beeindruckt von seiner Ruhe und Ausgewogenheit ausstrahlenden, Gesprächspartnern zugewandten Offenheit. Intensiver zusammengearbeitet haben wir während meiner Mitgliedschaft in der Kommission Deutscher Corporate Governance Kodex und besonders in der Zeit, in der ich den Vorsitz innehatte.

Die Deutsche Corporate Governance Kommission soll von der Bundesregierung unabhängig arbeiten können und der deutschen Wirtschaft mit dem von ihr entwickelten und laufend überprüften Kodex Hinweise geben, wie sich Unternehmensleitungen und Führungskräfte im Schwerpunkt börsennotierter Gesellschaften im Hinblick auf ihre Strukturen, auf Transparenz und bestimmte Entscheidungskriterien verhalten sollten. Sehr bewusst fördert der Kodex die Selbstregulierung der Unternehmen, die er mit Empfehlungen und Anregungen unterstützt. Der Kodex ergänzt den von den Unternehmen zu befolgenden Rechtsrahmen, lässt aber begründete Abweichungen von seinen Empfehlungen zu. Er baut - wie auch in anderen Ländern - auf dem Comply or Explain-Prinzip auf und gibt den Unternehmen damit Flexibilität und Entscheidungsfreiheit.

Prof. Seibert hat die Kommission von Anfang an vom Bundesministerium der Justiz aus „betreut“. Er war häufig Gast in den Sitzungen der Kommission und hat - sehr zurückhaltend, niemals fordernd oder vorschreibend - Hinweise und Rat gegeben. Die Empfehlungen der Kommission dürfen selbstverständlich nicht gegen rechtlich bindende Vorschriften verstoßen, können aber sehr wohl über sie hinausgehen. Die Gespräche mit Ulrich Seibert haben die Kommission darauf aufmerksam gemacht, wo Kollisionen im Rechtsbereich auftreten könnten und wo und mit welcher Zielrichtung gesetzgeberische Aktivitäten möglich wären. Auf der Grundlage fundierter Kenntnisse des Gesellschaftsrechts und der ministeriellen und parlamentarischen Abläufe waren seine Hinweise außerordentlich wertvoll. Sie dienten immer auch dazu, die Unabhängigkeit der Kommission zu wahren und abzusichern.

Das wurde besonders deutlich, wenn es personelle Wechsel in der Kommission und damit Erneuerungsbedarf gab. Er hat durch seinen Rat dazu beigetragen, dass die Vorschläge zur Bestellung neuer Kommissionsmitglieder ausgewogen unterschiedliche Erfahrungen und Kenntnisse berücksichtigten und so wahrscheinlich auch aus der Sicht der Bundesregierung angemessen waren. Im Ergebnis hat er mit dafür gesorgt, dass Ernennungen, die durch den Bundesminister oder die Bundesministerin der Justiz ausgesprochen wurden, niemals von den Vorschlägen aus der Kommission abwichen. Er hat gerade damit - eher unauffällig - die Unabhängigkeit der Kommission unterstützt. 
$\mathrm{Zu}$ diesen sachlichen Verdiensten kommt die sehr angenehme, freundliche, gründliche und zugewandte Persönlichkeit von Ulrich Seibert hinzu, mit dem man gern bei einem guten Essen und gutem Wein dann nicht nur „,̈̈ber das Geschäft", sondern auch über allgemeine politische und gesellschaftliche Entwicklungen, aber auch über Persönliches plaudern kann.

Ich bin Ihnen, lieber Herr Seibert, zu viel Dank verpflichtet und wünsche Ihnen alles Gute für noch viele schöne Jahre, die Sie hoffentlich wirklich genießen können.

Ihr

Manfred Gentz 